\title{
Mechanism of Acylation of Lithium Phenylacetylide with a Weinreb Amide
}

\author{
Bo Qu and David B. Collum* \\ Department of Chemistry and Chemical Biology \\ Baker Laboratory, Cornell University \\ Ithaca, New York 14853-1301
}

\section{Supporting Information}

\section{${ }^{6} \mathrm{Li}$ NMR Spectra and IR Kinetics Studies}

I. ${ }^{6} \mathrm{Li}$ NMR spectra recorded on $0.05 \mathrm{M}\left[{ }^{6} \mathrm{Li}\right] \mathrm{PhCCLi}$ and $0.06 \mathrm{M} 1$ at various temperatures.

II. $\quad{ }^{6} \mathrm{Li} \mathrm{NMR}$ spectrum of 5 .

III. $\quad J\left({ }^{6} \mathrm{Li},{ }^{13} \mathrm{C}\right)$-resolved NMR spectrum of $5 . \quad \mathrm{S} 3$

IV. $\quad{ }^{6} \mathrm{Li} \mathrm{NMR}{ }^{13} \mathrm{C}$ single frequency decoupled spectra of $5 . \quad \mathrm{S} 4$

V. Table of ${ }^{13} \mathrm{C}$ NMR resonances for different aggregates upon warming. $\quad$ S4

VI. $\quad{ }^{13} \mathrm{C}$ NMR spectrum of $5 . \quad$ S5

VII. $\quad{ }^{13} \mathrm{C},{ }^{13} \mathrm{C}$-COSY spectrum of $5 . \quad$ S5

$\begin{array}{lll}\text { VIII. } & { }^{6} \mathrm{Li} \text { NMR spectra of } 7 . & \text { S6 }\end{array}$

IX. $\quad J\left({ }^{6} \mathrm{Li},{ }^{13} \mathrm{C}\right)$-resolved NMR spectrum of $7 . \quad$ S6

X. $\quad{ }^{6} \mathrm{Li}$ NMR spectrum of uncharacterized aggregates upon warming to RT. $\quad$ S7

$\begin{array}{lll}\text { XI. } & \text { Plot of loss of carboxamide } 1 . & \text { S7 }\end{array}$

XII. Plot of $k_{\text {obsd }}$ vs [THF] for acylation of PhCCLi with $1 . \quad S 8$

$\begin{array}{ll}\text { XIII. Table of data for plot in Section XII } & \text { S8 }\end{array}$

XIV. Plot of $k_{\text {obsd }}$ Vs [PhCCLi] for acylation of PhCCLi with $1 . \quad$ S9

XV. Table of data for plot in Section XIV. $\quad$ S9

XVI. The Cartesian coordinates and energies for acylation of PhCCLi with 1 $\begin{array}{ll}\text { calculated at the B3LYP/6-31G(d) level. } & \text { S10 }\end{array}$

$\begin{array}{ll}\text { Experimental Section } & \text { S16 }\end{array}$ 


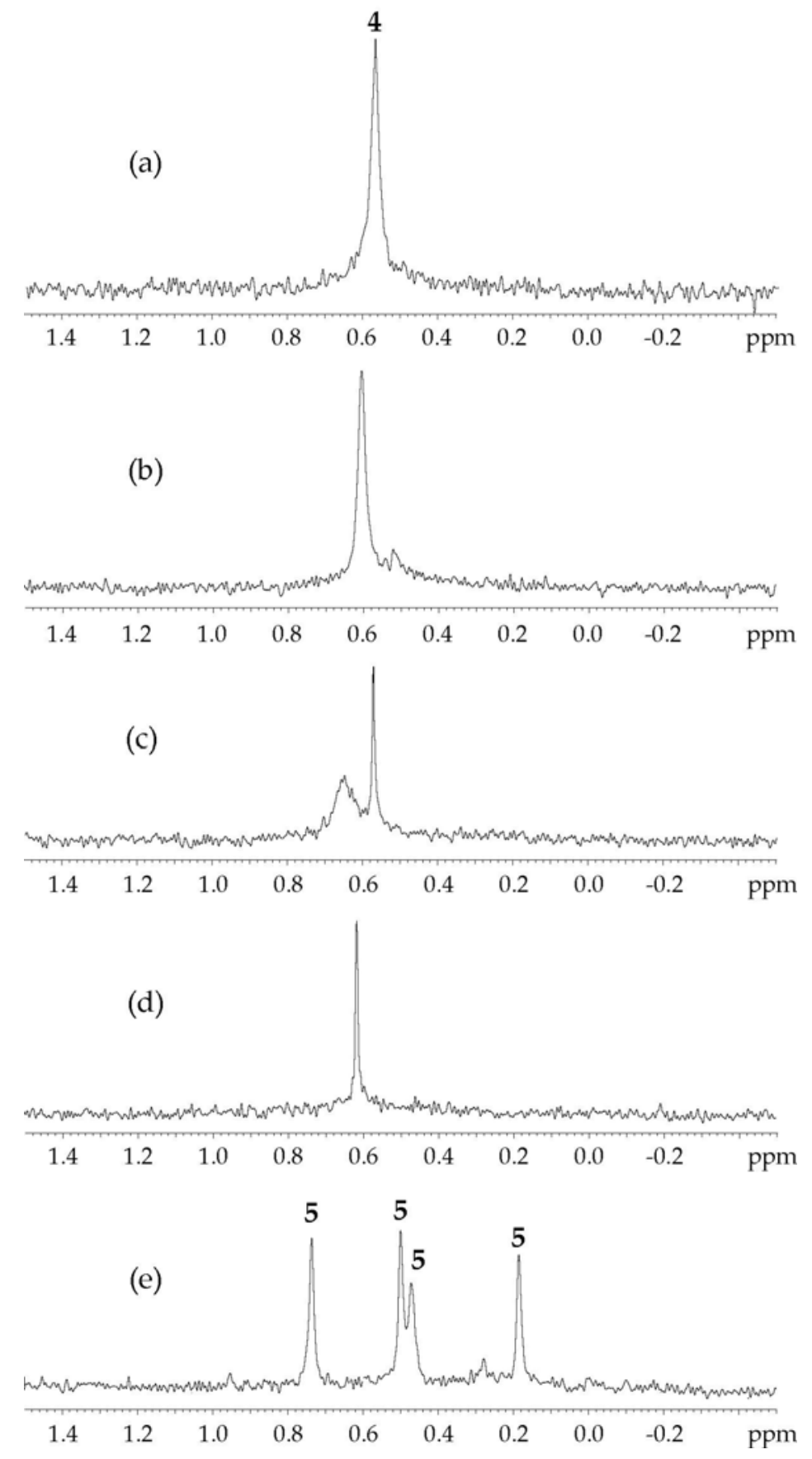

I. ${ }^{6} \mathrm{Li}$ NMR spectra* of a mixture of $0.05 \mathrm{M}\left[{ }^{6} \mathrm{Li}\right] \mathrm{PhCCLi} 4$ and $0.06 \mathrm{M} 1$ in $6.0 \mathrm{M}$ THF/pentane at: (a) $-90{ }^{\circ} \mathrm{C}$; (b) $-75{ }^{\circ} \mathrm{C}$; (c) $-55{ }^{\circ} \mathrm{C}$; (d) $-40{ }^{\circ} \mathrm{C}$; (e) back to $-90{ }^{\circ} \mathrm{C}$ after warming to $-40^{\circ} \mathrm{C}$, showing resonance of mixed aggregate 5 .

* ${ }^{6} \mathrm{Li}$ NMR spectra were recorded at $58.8 \mathrm{MHz}$. All other spectra in this Supporting Infomation were recorded at $73.7 \mathrm{MHz}$. 


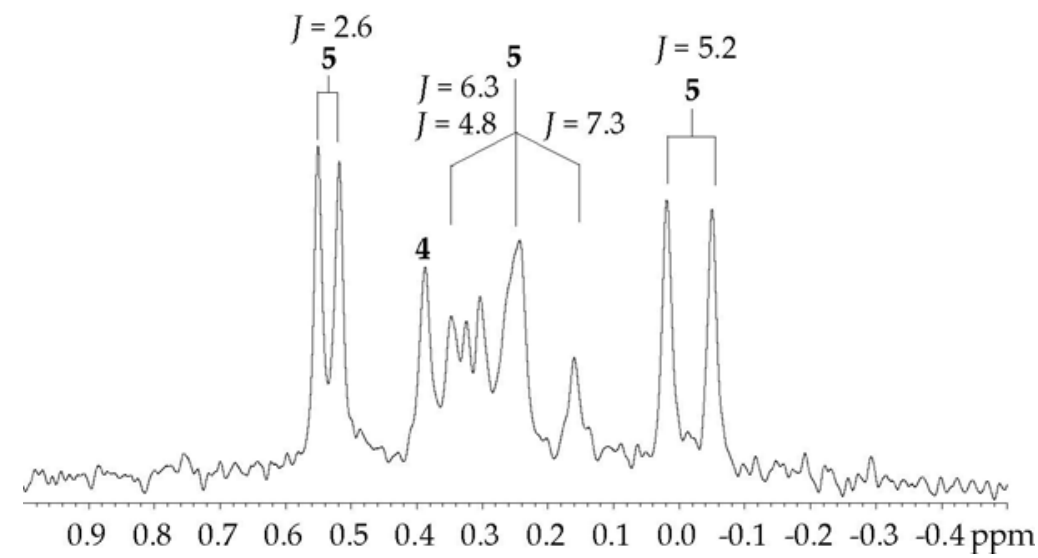

II. ${ }^{6} \mathrm{Li} \mathrm{NMR}$ spectrum of a mixture of $0.05 \mathrm{M}\left[{ }^{6} \mathrm{Li},{ }^{13} \mathrm{C}\right] \mathrm{PhCCLi}$ and $0.05 \mathrm{M} 1$ in $9.60 \mathrm{M}$ $\mathrm{THF} /$ pentane at $-110^{\circ} \mathrm{C}$, showing resonance of mixed aggregate 5 with unreacted PhCCLi 4. $J$ is in $\mathrm{Hz}$.

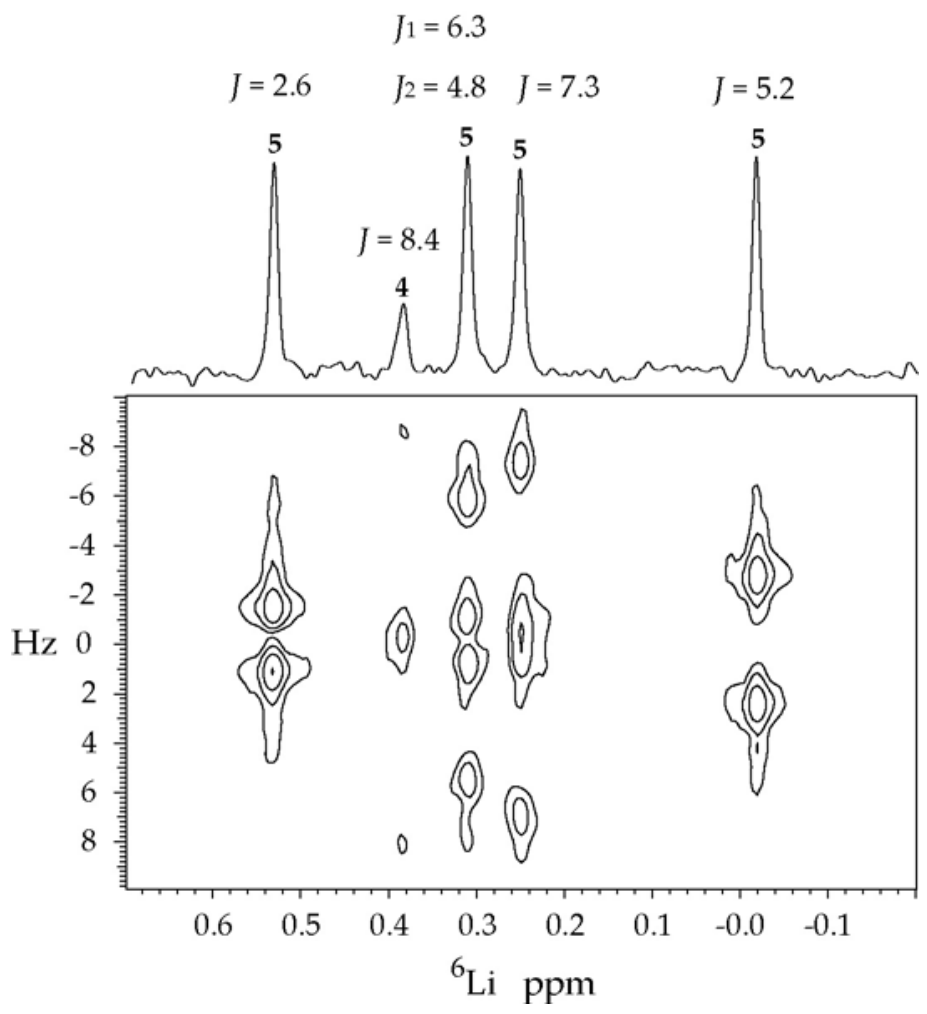

III. $J\left({ }^{6} \mathrm{Li},{ }^{13} \mathrm{C}\right)$-resolved spectrum of a mixture of $0.05 \mathrm{M}\left[{ }^{6} \mathrm{Li},{ }^{13} \mathrm{C}\right] \mathrm{PhCCLi}$ and $0.05 \mathrm{M} 1 \mathrm{in}$ 9.60 M THF/pentane at $-110{ }^{\circ} \mathrm{C} .{ }^{6} \mathrm{Li}$ spectrum is ${ }^{13} \mathrm{C}$ broad-band decoupled. $J$ is in $\mathrm{Hz}$. 

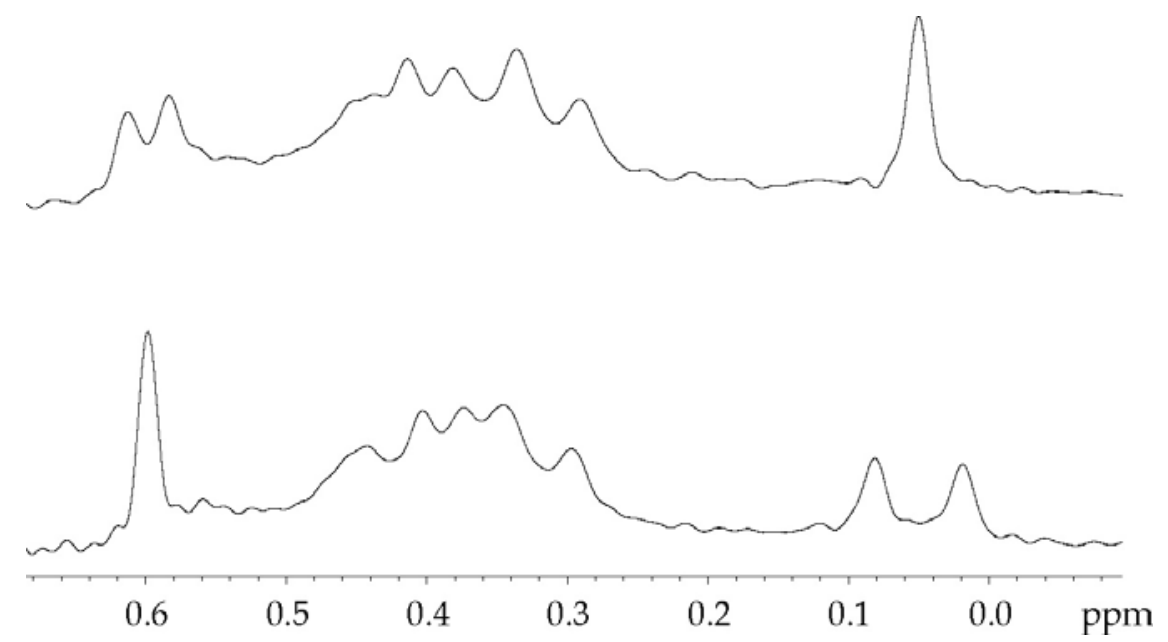

IV. ${ }^{6} \mathrm{Li} \mathrm{NMR}{ }^{13} \mathrm{C}$ single frequency decoupled spectra of 5 recorded on $0.05 \mathrm{M}$ $\left[{ }^{6} \mathrm{Li},{ }^{13} \mathrm{C}\right] \mathrm{PhCCLi}$ and $0.05 \mathrm{M} 1$ in $9.60 \mathrm{M} \mathrm{THF} /$ pentane at $-110{ }^{\circ} \mathrm{C}$.

V. Table of ${ }^{13} \mathrm{C}$ NMR resonances for different aggregates upon warming.

\begin{tabular}{|c|l|l|l|}
\hline \multirow{2}{*}{} & \multicolumn{3}{|c|}{${ }^{13} \mathrm{C}$ spectra } \\
\cline { 2 - 4 } & $\mathrm{R}-{ }^{13} \mathrm{C} \equiv \mathrm{C}-\mathrm{Ph}$ & $\mathrm{Li}-{ }^{13} \mathrm{C} \equiv \mathrm{C}-\mathrm{Ph}$ & $\begin{array}{l}\mathrm{Li}-{ }^{13} \mathrm{C} \equiv \mathrm{C}-\mathrm{Ph} \\
13 \mathrm{C}\left\{{ }^{6} \mathrm{Li}\right\}\end{array}$ \\
\hline $\mathbf{4}$ & na & 139.1 & $\begin{array}{l}139.1(\text { pentet}) \\
J=8.4 \mathrm{~Hz}\end{array}$ \\
\hline $\mathbf{5}$ & $95.1,97.2$ & $\begin{array}{l}135.1(\mathrm{~d}), 136.0(\mathrm{~d}) \\
J=5.5 \mathrm{~Hz}\end{array}$ & $\begin{array}{l}135.1(\text { sextet}) J=5.7 \mathrm{~Hz}^{*} \\
136.0(\mathrm{~m})\end{array}$ \\
\hline $\mathbf{7}$ & $93.3,95.7,96.5$ & 136.6 & $136.6(\mathrm{~m})$ \\
\hline $\begin{array}{c}\text { uncharacterized } \\
\text { aggregate after } \\
\text { warming to RT }\end{array}$ & 91.9 & na & na \\
\hline
\end{tabular}

* The peak shows incomplete multiple couplings. Theretically it should be triplet of heptet. 


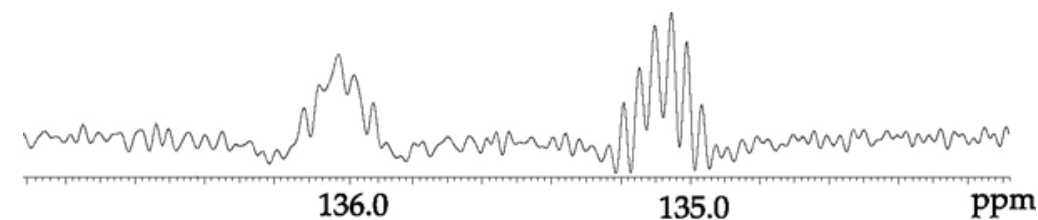

VI. ${ }^{13} \mathrm{C}$ NMR spectrum of 5 recorded on $0.05 \mathrm{M}\left[{ }^{6} \mathrm{Li},{ }^{13} \mathrm{C}\right] \mathrm{PhCCLi}$ and $0.05 \mathrm{M} 1$ in $9.60 \mathrm{M}$ $\mathrm{THF} /$ pentane at $-110{ }^{\circ} \mathrm{C}$ with sine bell resolution enhancement applied to show multiplet couplings.
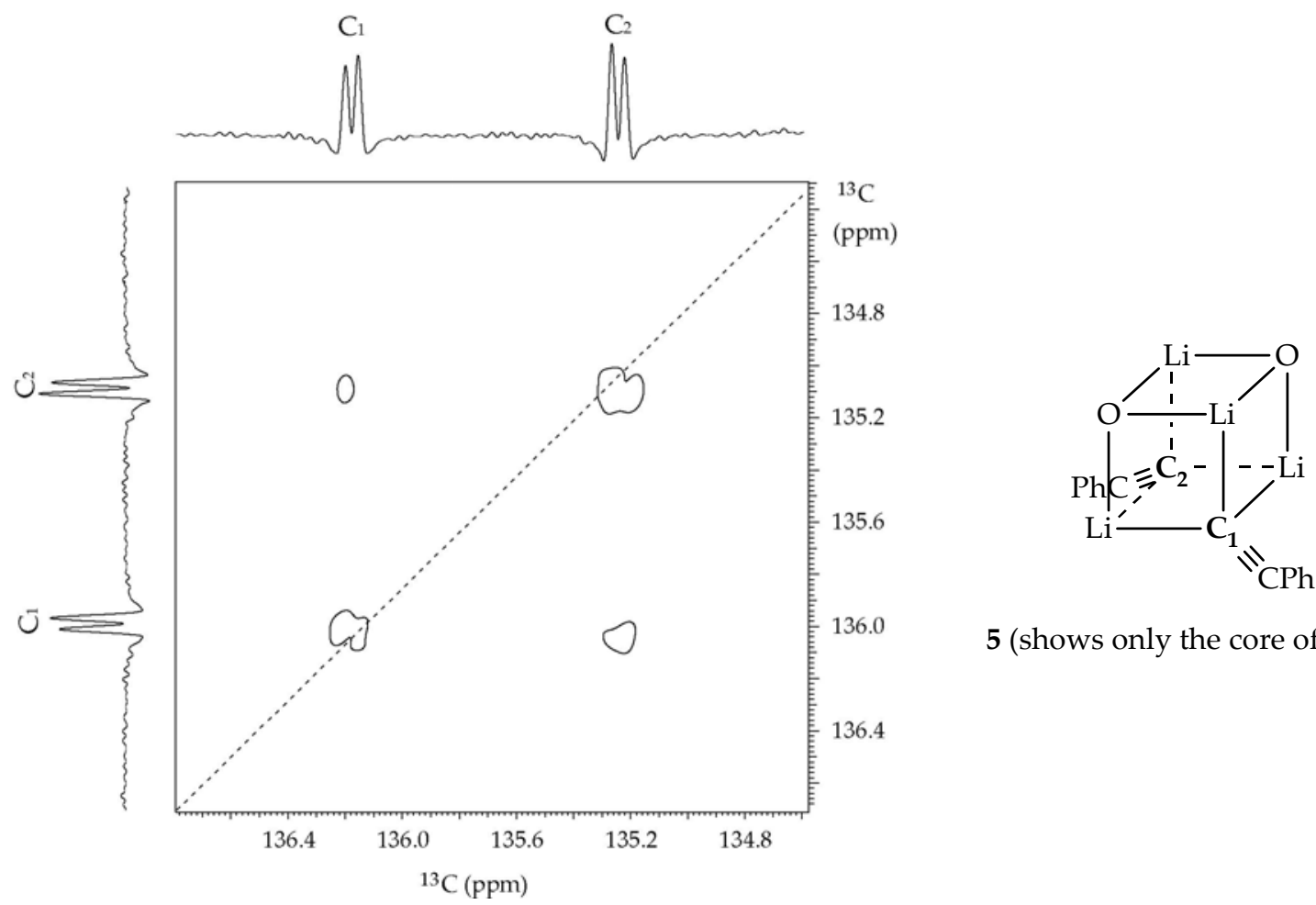

5 (shows only the core of tetramer)

VII. ${ }^{13} \mathrm{C},{ }^{13} \mathrm{C}$-COSY spectrum of 5 recorded on $0.05 \mathrm{M}\left[{ }^{6} \mathrm{Li},{ }^{13} \mathrm{C}\right] \mathrm{PhCCLi}$ and $0.05 \mathrm{M} \mathbf{1}$ in 9.60 M THF/pentane at $-110^{\circ} \mathrm{C}$, showing the coupling of $\mathrm{C}_{1}$ and $\mathrm{C}_{2} \cdot{ }^{13} \mathrm{C}$ spectrum is ${ }^{6} \mathrm{Li}$ broad-band decoupled with sine bell resolution enhancement applied to show the couplings. 


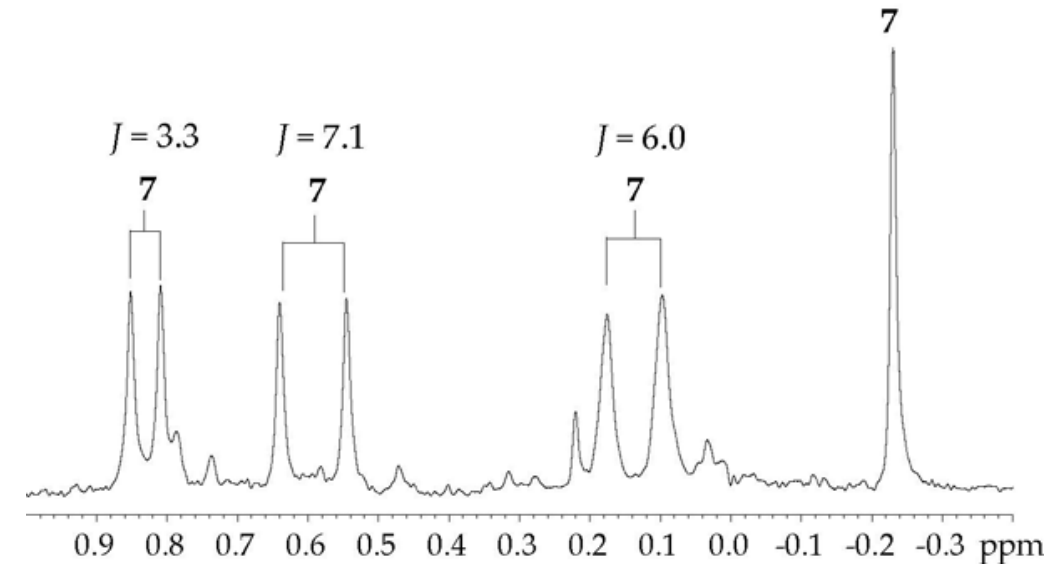

VIII. ${ }^{6} \mathrm{Li}$ NMR spectrum of 7 recorded on $0.05 \mathrm{M}\left[{ }^{6} \mathrm{Li},{ }^{13} \mathrm{C}\right] \mathrm{PhCCLi}$ and $0.05 \mathrm{M} \mathbf{1}$ in 9.60 $\mathrm{M} \mathrm{THF} /$ pentane at $-110^{\circ} \mathrm{C} . \mathrm{J}$ is in $\mathrm{Hz}$.

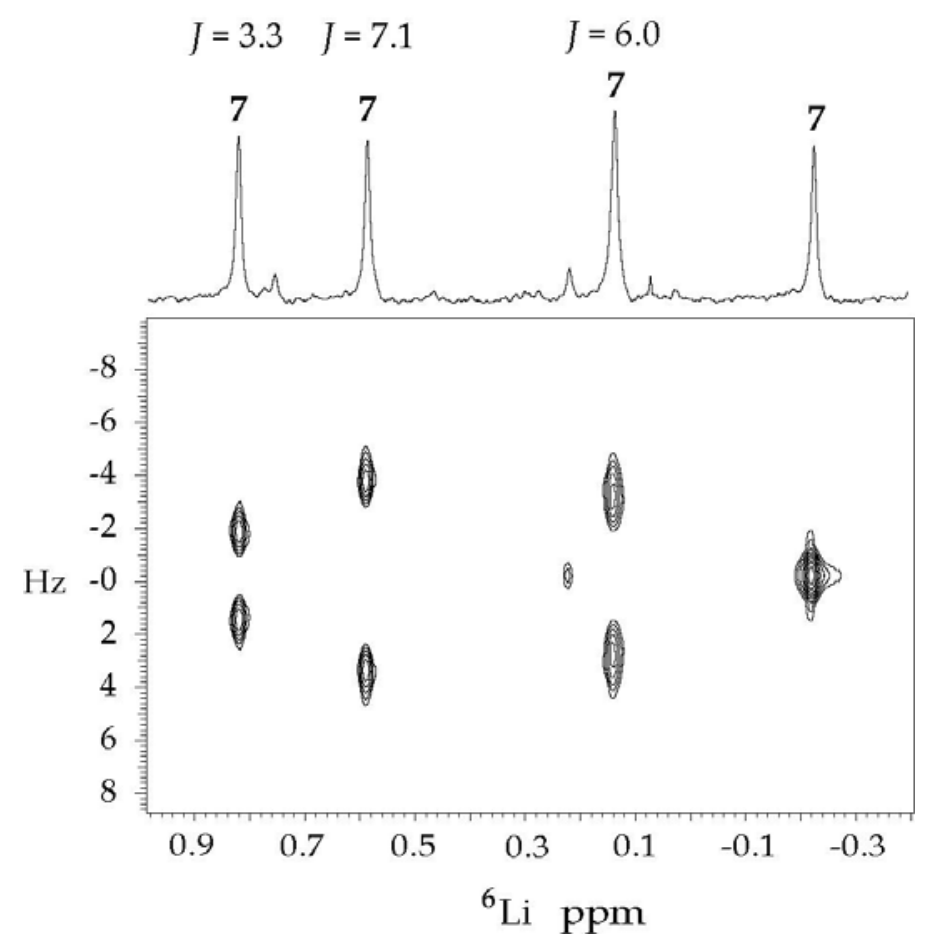

IX. $J\left({ }^{6} \mathrm{Li},{ }^{13} \mathrm{C}\right)$-resolved spectrum of 7 recorded on $0.05 \mathrm{M}\left[{ }^{6} \mathrm{Li},{ }^{13} \mathrm{C}\right]$ PhCCLi and 0.05 M 1 in $9.60 \mathrm{M} \mathrm{THF} /$ pentane at $-110{ }^{\circ} \mathrm{C} .{ }^{6} \mathrm{Li}$ spectrum is ${ }^{13} \mathrm{C}$ broad-band decoupled. $J$ is in $\mathrm{Hz}$. 


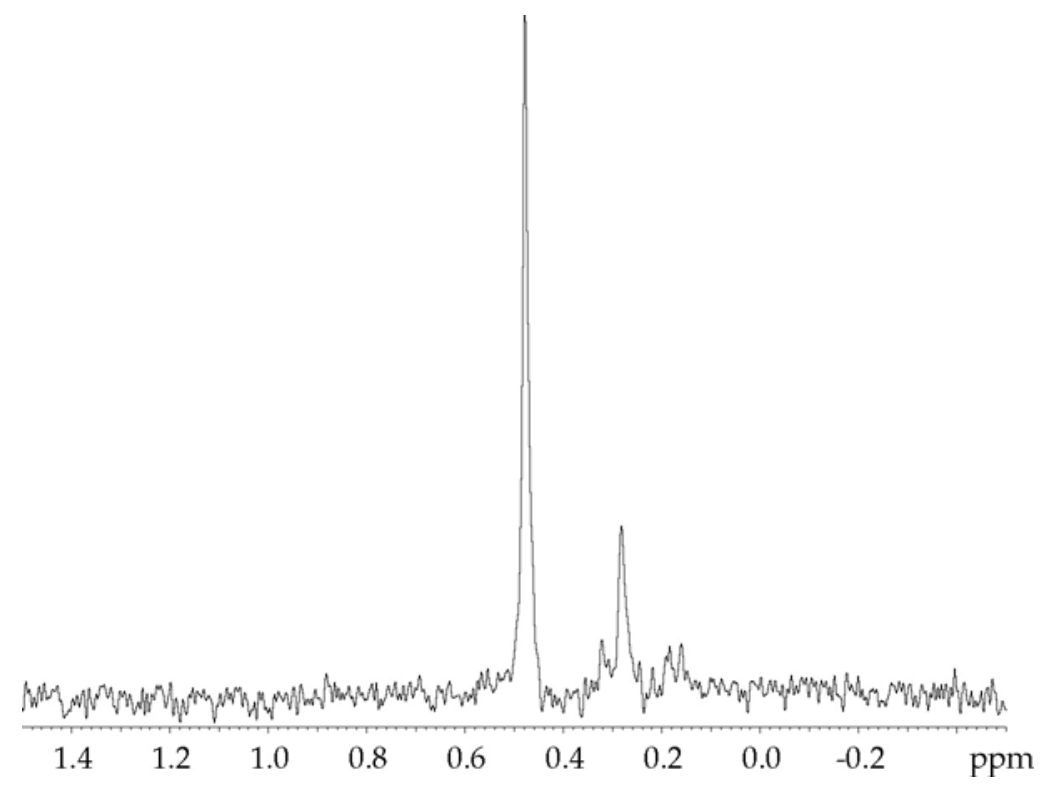

X. ${ }^{6} \mathrm{Li} \mathrm{NMR}$ spectrum recorded on a mixture $0.05 \mathrm{M}\left[{ }^{6} \mathrm{Li},{ }^{13} \mathrm{C}\right] \mathrm{PhCCLi}$ and $0.05 \mathrm{M} \mathbf{1}$ in 9.60 $\mathrm{M} \mathrm{THF} /$ pentane at $-110^{\circ} \mathrm{C}$, showing resonance of the uncharacterized aggregates upon further warming to RT.

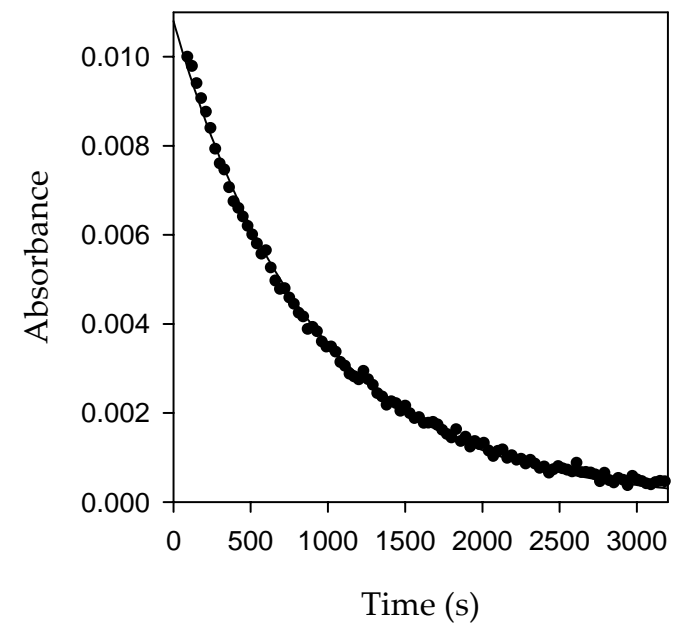

XI. ReactIR plot showing loss of carboxamide $1\left(1673 \mathrm{~cm}^{-1}\right)$ upon reaction with $0.10 \mathrm{M}$ $\mathrm{PhCCLi}$ in $4.0 \mathrm{M} \mathrm{THF} /$ pentane at $-50^{\circ} \mathrm{C}$. 


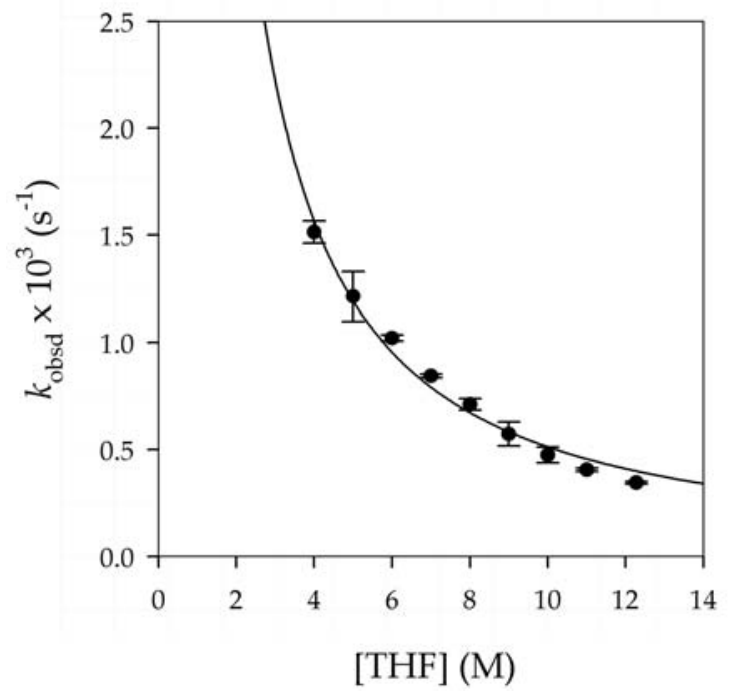

XII. Plot of $k_{\text {obsd }}$ Vs [THF] for acylation of PhCCLi $(0.10 \mathrm{M})$ with $\mathbf{1}(0.005 \mathrm{M})$ in pentane cosolvent at $-50^{\circ} \mathrm{C}$. The curve depicts an unweighted least-squares fit to $k_{\mathrm{obsd}}=a[\mathrm{THF}]^{n}$ ( $a$ $\left.=8.5 \pm 0.9 \times 10^{-3}, n=-1.22 \pm 0.06\right)$.

XIII. Table of data for plot in Section XII.

$\begin{array}{lllll}{[\mathrm{THF}](\mathrm{M})} & k_{\mathrm{obsd}} 1\left(\mathrm{~s}^{-1}\right) & k_{\mathrm{obsd}} 2\left(\mathrm{~s}^{-1}\right) & k_{\mathrm{obsd}} 3\left(\mathrm{~s}^{-1}\right) & k_{\mathrm{obsd}}(\mathrm{av})\left(\mathrm{s}^{-1}\right) \\ 4.0 & 0.00150 \pm 4 \mathrm{E}-5 & 0.00157 \pm 1 \mathrm{E}-5 & 0.00147 \pm 1 \mathrm{E}-5 & 0.00151 \pm 5 \mathrm{E}-5 \\ 5.0 & 0.00133 \pm 3 \mathrm{E}-5 & 0.00110 \pm 1 \mathrm{E}-5 & 0.00121 \pm 1 \mathrm{E}-5 & 0.0012 \pm 1 \mathrm{E}-4 \\ 6.0 & 0.00103 \pm 5 \mathrm{E}-5 & 0.00101 \pm 2 \mathrm{E}-5 & & 0.00102 \pm 1 \mathrm{E}-5 \\ 7.0 & 0.000850 \pm 7 \mathrm{E}-6 & 0.000838 \pm 4 \mathrm{E}-6 & 0.00084 \pm 1 \mathrm{E}-5 \\ 8.0 & 0.00073 \pm 3 \mathrm{E}-5 & 0.000692 \pm 4 \mathrm{E}-6 & & 0.00071 \pm 3 \mathrm{E}-5 \\ 9.0 & 0.000533 \pm 4 \mathrm{E}-6 & 0.000612 \pm 4 \mathrm{E}-6 & 0.00057 \pm 5 \mathrm{E}-5 \\ 10.0 & 0.00050 \pm 1 \mathrm{E}-5 & 0.000448 \pm 3 \mathrm{E}-6 & & 0.00047 \pm 4 \mathrm{E}-5 \\ 11.0 & 0.000400 \pm 2 \mathrm{E}-6 & 0.000411 \pm 2 \mathrm{E}-6 & & 0.000406 \pm 8 \mathrm{E}-6 \\ 12.27 & 0.000340 \pm 5 \mathrm{E}-6 & 0.000349 \pm 2 \mathrm{E}-6 & & 0.000345 \pm 6 \mathrm{E}-6\end{array}$




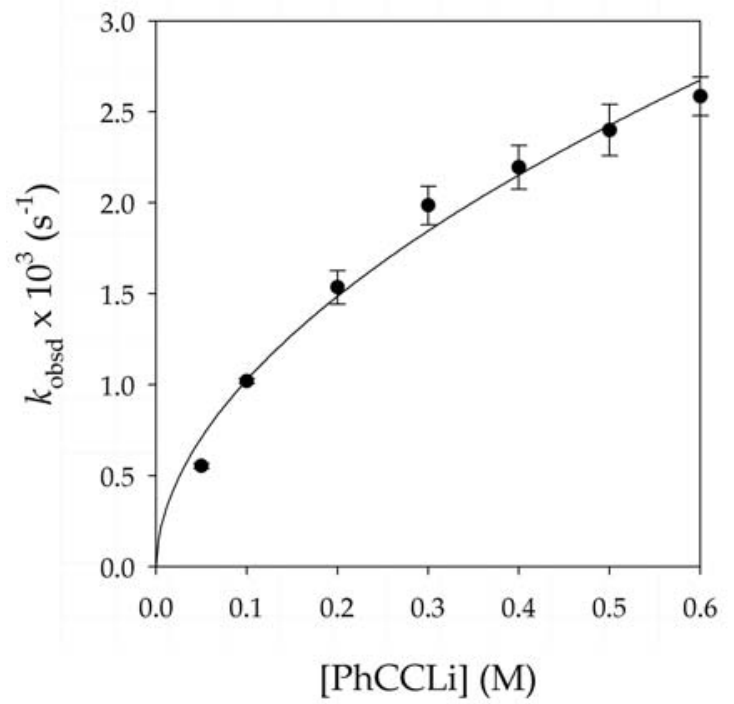

XIV. Plot of $k_{\text {obsd }}$ Vs [PhCCLi] for acylation of PhCCLi with $1(0.005 \mathrm{M})$ in $6.0 \mathrm{M}$ THF and pentane cosolvent at $-50{ }^{\circ} \mathrm{C}$. The curve depicts an unweighted least-squares fit to $k_{\mathrm{obsd}}$ $=a[\mathrm{PhCCLi}]^{b}\left(a=3.5 \pm 0.2 \times 10^{-3}, b=0.53 \pm 0.04\right)$.

XV. Table of data for plot in Section XIV.

$\begin{array}{llll}{[\mathrm{PhCCLi}](\mathrm{M})} & k_{\text {obsd }} 1\left(\mathrm{~s}^{-1}\right) & k_{\text {obsd }} 2\left(\mathrm{~s}^{-1}\right) & k_{\text {obsd }}(\mathrm{av})\left(\mathrm{s}^{-1}\right) \\ 0.05 & 0.000542 \pm 4 \mathrm{E}-6 & 0.000563 \pm 5 \mathrm{E}-6 & 0.00055 \pm 1 \mathrm{E}-5 \\ 0.10 & 0.00103 \pm 5 \mathrm{E}-5 & 0.00101 \pm 2 \mathrm{E}-5 & 0.00102 \pm 1 \mathrm{E}-5 \\ 0.20 & 0.00147 \pm 2 \mathrm{E}-5 & 0.00160 \pm 2 \mathrm{E}-5 & 0.00154 \pm 9 \mathrm{E}-5 \\ 0.30 & 0.00191 \pm 3 \mathrm{E}-5 & 0.00206 \pm 3 \mathrm{E}-5 & 0.0020 \pm 1 \mathrm{E}-4 \\ 0.40 & 0.00211 \pm 4 \mathrm{E}-5 & 0.00228 \pm 5 \mathrm{E}-5 & 0.0022 \pm 1 \mathrm{E}-4 \\ 0.50 & 0.00230 \pm 3 \mathrm{E}-5 & 0.00248 \pm 4 \mathrm{E}-5 & 0.0024 \pm 1 \mathrm{E}-4 \\ 0.60 & 0.00251 \pm 4 \mathrm{E}-5 & 0.00266 \pm 6 \mathrm{E}-5 & 0.0026 \pm 1 \mathrm{E}-4\end{array}$


XVI. The Cartesian coordinates and energies for the reaction of Weinreb Amide 1 with Lithium Phenylacetylide calculated at the B3LYP/6-31G(d) level (Energy values and coordinates are in Hartrees and Angstroms, respectively).

\section{Dimethyl ether}

SCF Done: $\mathrm{E}(\mathrm{RB}+\mathrm{HF}-\mathrm{LYP})=$

Zero-point correction $=$

Thermal correction to Energy=

Thermal correction to Enthalpy=

Thermal correction to Gibbs Free Energy=

Sum of electronic and zero-point Energies=

Sum of electronic and thermal Energies=

Sum of electronic and thermal Enthalpies=

Sum of electronic and thermal Free Energies=

-155.025044225
0.080306 (Hartree/Particle)
0.084599
0.085544
0.054941
-154.944738
-154.940445
-154.939501
-154.970103

\begin{tabular}{cccccc} 
Center & Atomic & Atomic & \multicolumn{3}{c}{ Coordinates } \\
Number & Number & Type & $\mathrm{X}$ & $\mathrm{Y}$ & $\mathrm{Z}$ \\
\hline 1 & 6 & 0 & -1.170929 & 0.195252 & -0.000006 \\
2 & 8 & 0 & -0.000014 & -0.589896 & 0.000002 \\
3 & 6 & 0 & 1.170935 & 0.195242 & 0.000003 \\
4 & 1 & 0 & -1.232061 & 0.839934 & -0.892752 \\
5 & 1 & 0 & -2.021904 & -0.491258 & -0.000344 \\
6 & 1 & 0 & -1.232447 & 0.839444 & 0.893087 \\
7 & 1 & 0 & 1.232215 & 0.839800 & 0.892842 \\
8 & 1 & 0 & 2.021897 & -0.491289 & 0.000160 \\
9 & 1 & 0 & 1.232371 & 0.839569 & -0.892992 \\
\hline & & & & & \\
\hline & & 1 & 250.0383 & 415.2796
\end{tabular}




\section{Weinreb amide 1}

SCF Done: $\mathrm{E}(\mathrm{RB}+\mathrm{HF}-\mathrm{LYP})=$

Zero-point correction $=$

Thermal correction to Energy=

Thermal correction to Enthalpy=

Thermal correction to Gibbs Free Energy=

Sum of electronic and zero-point Energies=

Sum of electronic and thermal Energies $=$

Sum of electronic and thermal Enthalpies=

Sum of electronic and thermal Free Energies=
$-362.985543527$

0.134747 (Hartree/Particle)

0.143392

0.144337

0.101792

$-362.850797$

$-362.842151$

$-362.841207$

$-362.883752$

\begin{tabular}{ccccccc} 
Center & \multicolumn{3}{c}{ Atomic } & Atomic & \multicolumn{3}{c}{ Coordinates } \\
Number & Number & Type & $\mathrm{X}$ & $\mathrm{Y}$ & $\mathrm{Z}$ \\
\hline & \multicolumn{3}{c}{ - } & & & \\
1 & 6 & 0 & 2.269916 & -0.099961 & 0.283493 \\
2 & 6 & 0 & 0.845791 & -0.525003 & -0.052876 \\
3 & 8 & 0 & 0.558622 & -1.677713 & -0.328811 \\
4 & 7 & 0 & -0.122940 & 0.465609 & 0.060615 \\
5 & 6 & 0 & 0.113906 & 1.892456 & -0.086016 \\
6 & 8 & 0 & -1.367898 & 0.133757 & -0.510453 \\
7 & 6 & 0 & -2.195480 & -0.531670 & 0.446628 \\
8 & 1 & 0 & 2.874543 & -1.006180 & 0.338166 \\
9 & 1 & 0 & 2.684284 & 0.554174 & -0.492731 \\
10 & 1 & 0 & 2.329036 & 0.431183 & 1.239522 \\
11 & 1 & 0 & 0.133687 & 2.193272 & -1.142381 \\
12 & 1 & 0 & -0.689849 & 2.435834 & 0.417893 \\
13 & 1 & 0 & 1.061403 & 2.160334 & 0.383545 \\
14 & 1 & 0 & -3.152692 & -0.673902 & -0.062009 \\
15 & 1 & 0 & -1.770412 & -1.502081 & 0.717299 \\
16 & 1 & 0 & -2.340011 & 0.084820 & 1.343120
\end{tabular}


$\begin{array}{cccc} & 1 & 2 & 3 \\ \text { Frequencies -- } & 96.3286 & 110.9917 & 142.1267\end{array}$

\section{Lithium phenylacetylide dimer $(\mathrm{PhCCLi})_{2} \cdot\left(\mathrm{Me}_{2} \mathrm{O}\right)_{4}$}

SCF Done: $\mathrm{E}(\mathrm{RB}+\mathrm{HF}-\mathrm{LYP})=$

Zero-point correction $=$

Thermal correction to Energy=

Thermal correction to Enthalpy=

Thermal correction to Gibbs Free Energy=

Sum of electronic and zero-point Energies $=$

Sum of electronic and thermal Energies=

Sum of electronic and thermal Enthalpies=

Sum of electronic and thermal Free Energies=
$-1250.93775564$

0.530908 (Hartree/Particle)

0.571599

0.572544

0.442905

$-1250.406847$

$-1250.366156$

$-1250.365212$

$-1250.494851$

\begin{tabular}{cccccc} 
Center & \multicolumn{2}{c}{ Atomic } & Atomic & \multicolumn{3}{c}{ Coordinates } \\
Number & Number & Type & $\mathrm{X}$ & $\mathrm{Y}$ & $\mathrm{Z}$ \\
& & & & & \\
- & 6 & 0 & -3.010047 & 0.028976 & 0.005445 \\
1 & 6 & 0 & -1.771641 & 0.061733 & 0.008779 \\
3 & 3 & 0 & 0.000052 & 1.316565 & -0.000008 \\
4 & 3 & 0 & -0.000329 & -1.148892 & 0.000049 \\
5 & 6 & 0 & 1.771416 & 0.061093 & -0.007811 \\
6 & 6 & 0 & 3.009831 & 0.028585 & -0.005433 \\
7 & 6 & 0 & 4.441514 & -0.004349 & -0.006372 \\
8 & 6 & 0 & 5.169247 & 0.009676 & -1.215494 \\
9 & 6 & 0 & 6.561670 & -0.025251 & -1.214130 \\
10 & 6 & 0 & 7.266575 & -0.074551 & -0.008857 \\
11 & 6 & 0 & 6.562339 & -0.088359 & 1.197693 \\
12 & 6 & 0 & 5.169936 & -0.053903 & 1.201335 \\
13 & 8 & 0 & -0.000674 & -2.313389 & -1.625844 \\
14 & 6 & 0 & -1.186465 & -2.890680 & -2.153073
\end{tabular}




\begin{tabular}{|c|c|c|c|c|c|}
\hline 15 & 6 & 0 & 1.187289 & -2.755444 & -2.268157 \\
\hline 16 & 8 & 0 & -0.000343 & -2.316204 & 1.624172 \\
\hline 17 & 6 & 0 & -1.187494 & -2.750102 & 2.273547 \\
\hline 18 & 6 & 0 & 1.186334 & -2.884482 & 2.159210 \\
\hline 19 & 8 & 0 & 0.107312 & 2.603466 & 1.520654 \\
\hline 20 & 6 & 0 & -1.059178 & 3.367049 & 1.805885 \\
\hline 21 & 6 & 0 & 0.789993 & 2.169200 & 2.693291 \\
\hline 22 & 8 & 0 & -0.106571 & 2.602375 & -1.521470 \\
\hline 23 & 6 & 0 & -0.789161 & 2.167422 & -2.693931 \\
\hline 24 & 6 & 0 & 1.060124 & 3.365507 & -1.807054 \\
\hline 25 & 6 & 0 & -4.441730 & -0.004011 & 0.005365 \\
\hline 26 & 6 & 0 & -5.169257 & -0.054673 & -1.202835 \\
\hline 27 & 6 & 0 & -6.561661 & -0.089123 & -1.200184 \\
\hline 28 & 6 & 0 & -7.266787 & -0.074219 & 0.005834 \\
\hline 29 & 6 & 0 & -6.562773 & -0.023826 & 1.211583 \\
\hline 30 & 6 & 0 & -5.170351 & 0.011106 & 1.213939 \\
\hline 31 & 1 & 0 & -1.342820 & -3.829809 & 2.131788 \\
\hline 32 & 1 & 0 & -2.009511 & -2.188013 & 1.826778 \\
\hline 33 & 1 & 0 & -1.138576 & -2.536222 & 3.350971 \\
\hline 34 & 1 & 0 & -2.021890 & -2.408467 & -1.642278 \\
\hline 35 & 1 & 0 & -1.261195 & -2.702534 & -3.233817 \\
\hline 36 & 1 & 0 & -1.209186 & -3.976500 & -1.978541 \\
\hline 37 & 1 & 0 & 1.785440 & 2.771591 & -2.378959 \\
\hline 38 & 1 & 0 & 0.799749 & 4.271151 & -2.373264 \\
\hline 39 & 1 & 0 & 1.499286 & 3.645156 & -0.847944 \\
\hline 40 & 1 & 0 & 1.617561 & 1.543171 & 2.356041 \\
\hline 41 & 1 & 0 & 1.169146 & 3.032519 & 3.258641 \\
\hline 42 & 1 & 0 & 0.120913 & 1.581704 & 3.338022 \\
\hline 43 & 1 & 0 & -7.100088 & -0.127440 & -2.144166 \\
\hline 44 & 1 & 0 & -4.622598 & -0.065451 & -2.141539 \\
\hline 45 & 1 & 0 & -4.624168 & 0.052183 & 2.152018 \\
\hline 46 & 1 & 0 & -7.102215 & -0.010954 & 2.155682 \\
\hline
\end{tabular}




\begin{tabular}{|c|c|c|c|c|c|}
\hline 47 & 1 & 0 & -8.353278 & -0.101021 & 0.006021 \\
\hline 48 & 1 & 0 & 7.100418 & -0.013239 & -2.158636 \\
\hline 49 & 1 & 0 & 4.622376 & 0.049896 & -2.153211 \\
\hline 50 & 1 & 0 & 4.623974 & -0.063824 & 2.140454 \\
\hline 51 & 1 & 0 & 7.101456 & -0.125823 & 2.141315 \\
\hline 52 & 1 & 0 & 8.353065 & -0.101358 & -0.009828 \\
\hline 53 & 1 & 0 & 1.260358 & -2.682260 & 3.237450 \\
\hline 54 & 1 & 0 & 1.210978 & -3.972411 & 1.998692 \\
\hline 55 & 1 & 0 & -1.617019 & 1.541951 & -2.356364 \\
\hline 56 & 1 & 0 & -1.167884 & 3.030448 & -3.260014 \\
\hline 57 & 1 & 0 & -0.120165 & 1.579160 & -3.338036 \\
\hline 58 & 1 & 0 & -1.784579 & 2.773641 & 2.378213 \\
\hline 59 & 1 & 0 & -0.798536 & 4.272973 & 2.371522 \\
\hline 60 & 1 & 0 & -1.498365 & 3.646220 & 0.846649 \\
\hline 61 & 1 & 0 & 2.021141 & -2.407333 & 1.642715 \\
\hline 62 & 1 & 0 & 1.342727 & -3.833197 & -2.112251 \\
\hline 63 & 1 & 0 & 1.139549 & -2.555579 & -3.348320 \\
\hline 64 & 1 & 0 & 2.008639 & -2.187588 & -1.827470 \\
\hline & & 1 & 2 & & 3 \\
\hline \multicolumn{2}{|c|}{ Frequencies -- } & 7.1050 & \multicolumn{2}{|c|}{8.2535} & 12.6860 \\
\hline
\end{tabular}

\section{Transition Structure}

SCF Done: $\mathrm{E}(\mathrm{RB}+\mathrm{HF}-\mathrm{LYP})=$

Zero-point correction=

Thermal correction to Energy=

Thermal correction to Enthalpy=

Thermal correction to Gibbs Free Energy=

Sum of electronic and zero-point Energies $=$

Sum of electronic and thermal Energies=

Sum of electronic and thermal Enthalpies=
$-833.378121098$
0.318725 (Hartree/Particle)
0.341000
0.341945
0.262301
$-833.059396$
$-833.037121$
$-833.036176$ 


\begin{tabular}{|c|c|c|c|c|c|}
\hline Center & Atomic & Atomic & \multicolumn{3}{|c|}{ Coordinates } \\
\hline Number & Number & Type & $X$ & $\mathrm{Y}$ & $\mathrm{Z}$ \\
\hline 1 & 3 & 0 & 1.643978 & 0.833698 & -0.061057 \\
\hline 2 & 8 & 0 & 2.271320 & -0.253138 & 1.333890 \\
\hline 3 & 6 & 0 & 1.570915 & -1.185012 & 0.827877 \\
\hline 4 & 7 & 0 & 1.981381 & -1.741733 & -0.451160 \\
\hline 5 & 8 & 0 & 2.635132 & -0.715884 & -1.215893 \\
\hline 6 & 6 & 0 & 4.053838 & -0.578870 & -0.986252 \\
\hline 7 & 6 & 0 & 2.667139 & -3.022335 & -0.502212 \\
\hline 8 & 6 & 0 & 0.891909 & -2.198418 & 1.734890 \\
\hline 9 & 6 & 0 & -0.125155 & -0.253238 & 0.133776 \\
\hline 10 & 6 & 0 & -1.356054 & -0.214549 & 0.068264 \\
\hline 11 & 6 & 0 & -2.780150 & -0.156270 & -0.036804 \\
\hline 12 & 6 & 0 & -3.568235 & 0.294934 & 1.042587 \\
\hline 13 & 6 & 0 & -4.956084 & 0.346362 & 0.938878 \\
\hline 14 & 6 & 0 & -5.590319 & -0.048238 & -0.241953 \\
\hline 15 & 6 & 0 & -4.823266 & -0.497081 & -1.320289 \\
\hline 16 & 6 & 0 & -3.435256 & -0.552387 & -1.221696 \\
\hline 17 & 8 & 0 & 1.938266 & 2.730509 & -0.172432 \\
\hline 18 & 6 & 0 & 2.291634 & 3.363193 & 1.058859 \\
\hline 19 & 6 & 0 & 1.154891 & 3.562914 & -1.025594 \\
\hline 20 & 1 & 0 & 0.217203 & 3.850861 & -0.533036 \\
\hline 21 & 1 & 0 & 0.929741 & 2.983920 & -1.923387 \\
\hline 22 & 1 & 0 & 1.717574 & 4.464527 & -1.301414 \\
\hline 23 & 1 & 0 & 2.833552 & 2.624454 & 1.651961 \\
\hline 24 & 1 & 0 & 2.931636 & 4.234955 & 0.869445 \\
\hline 25 & 1 & 0 & 1.391915 & 3.680660 & 1.602156 \\
\hline 26 & 1 & 0 & 4.336385 & 0.313628 & -1.551869 \\
\hline 27 & 1 & 0 & 4.270156 & -0.442206 & 0.075058 \\
\hline
\end{tabular}




\begin{tabular}{|c|c|c|c|c|c|}
\hline 28 & 1 & 0 & 4.601849 & -1.437711 & -1.389115 \\
\hline 29 & 1 & 0 & 3.620012 & -3.065017 & 0.050018 \\
\hline 30 & 1 & 0 & 1.995325 & -3.777860 & -0.089180 \\
\hline 31 & 1 & 0 & 2.847822 & -3.272631 & -1.551644 \\
\hline 32 & 1 & 0 & 1.664813 & -2.809897 & 2.220538 \\
\hline 33 & 1 & 0 & 0.351832 & -1.652721 & 2.508642 \\
\hline 34 & 1 & 0 & 0.195066 & -2.842064 & 1.194665 \\
\hline 35 & 1 & 0 & -5.309864 & -0.806723 & -2.241938 \\
\hline 36 & 1 & 0 & -2.836506 & -0.902670 & -2.057203 \\
\hline 37 & 1 & 0 & -3.072943 & 0.600825 & 1.959497 \\
\hline 38 & 1 & 0 & -5.546213 & 0.695683 & 1.782588 \\
\hline \multirow[t]{2}{*}{39} & 1 & 0 & -6.673540 & -0.006892 & -0.321000 \\
\hline & & 1 & 2 & & 3 \\
\hline \multicolumn{2}{|c|}{ requencies-- } & -276.2004 & 10.26 & 634 & 4.276 \\
\hline
\end{tabular}

\section{Experimental Section}

Reagents and Solvents. THF and pentane were vacuum-transferred from degassed blue or purple stills containing sodium benzophenone ketyl. The pentane still contained $1 \%$ tetraglyme to dissolve the ketyl. Air- and moisture-sensitive materials were manipulated using vacuum line and syringe techniques. Weinreb amide $\mathbf{1}$ was prepared from cyclohexanecarboxaldehyde and N-methyl-N-methoxylhydroxylamine hydrochloride using literature procedures. $\left[{ }^{6} \mathrm{Li}\right] \mathrm{PhCCLi}$ and $\left[{ }^{6} \mathrm{Li},{ }^{13} \mathrm{C}\right] \mathrm{PhCCLi}$ were prepared and recrystallized in pentane using recrystallized [ $\left.{ }^{6} \mathrm{Li}\right] \mathrm{LiHMDS}$ and commercially available phenylacetylene and $\left[{ }^{13} \mathrm{C}-1\right]$ phenylacetylene. The phenylacetylenes were vacuum-transferred from $4 \AA$ molecular sieves.

NMR Spectroscopic Analyses. All tubes were prepared under helium and stored in a liquid nitrogen bath prior to analysis. Tubes were prepared using stock solutions and sealed under partial vacuum. Standard ${ }^{6} \mathrm{Li}$ and ${ }^{13} \mathrm{C}$ NMR spectra were recorded on a $500 \mathrm{MHz}$ 
spectrometer at 73.7 and $125.0 \mathrm{~Hz}$ respectively. The ${ }^{6} \mathrm{Li}$ and resonances were referenced to $0.3 \mathrm{M}\left[{ }^{6} \mathrm{Li}\right] \mathrm{LiCl} / \mathrm{MeOH}$ at $-90{ }^{\circ} \mathrm{C}(0.0 \mathrm{ppm})$. The spectra were recorded with a threechannel probe designed to accommodate lithium and carbon pulses. The tube was transferred from the liquid nitrogen bath to a $-78{ }^{\circ} \mathrm{C}$ bath to thaw the solution, then quickly transferred into the spectrometer and shimmed off the proton spectrum.

Rate Studies by In Situ ReactIR Spectroscopy. IR spectra were recorded using an in situ IR spectrometer fitted with a 30-bounce silicon-tipped probe. The IR probe was inserted through a nylon adapter and FETFE O-ring seal into a cylindrical flask fitted with a magnetic stir bar and T-joint. The T-joint was fitted with a nitrogen line and septum for injections. The flask was heated under full-vacuum and flushed twice with nitrogen. PhCCLi was weighted in glove box and dissolved in THF before syringe-transferred to the IR vessel. Pentane was added to the vessel to make the total volume $10 \mathrm{~mL}$. The solution mixture was cooled to $-50^{\circ} \mathrm{C}$ in a thermostatted bath for 25 minutes. A background spectrum was recorded, followed by addition of $0.05 \mathrm{~mL}$ of a $1.0 \mathrm{M}$ stock solution of $\mathbf{1}$ while stirring. Spectra were recorded every 30 seconds for 5 half-lives. The rates of acylation of lithium penylacetylide with Weinreb amide $\mathbf{1}$ were monitored by following the loss of $1\left(1673 \mathrm{~cm}^{-1}\right)$.

The disappearance of the amide $\mathbf{1}$ was fit to the equation $[\mathrm{A}] /\left[\mathrm{A}_{0}\right]=\mathrm{a}^{*} \mathrm{e}^{-k_{\text {obsa }} \mathrm{t}}+\mathrm{c}$. Where $[\mathrm{A}]$ is the concentration of $\mathbf{1}$ at time " $\mathrm{t}$ " and $\left[\mathrm{A}_{0}\right]$ is the concentration of $\mathbf{1}$ at $\mathrm{t}=0$. Term " $\mathrm{c}$ " is a measure of the amount of amide remaining at $\mathrm{t}=\infty$, which is typically a positive number at $\leq 5 \%$ of $\left[\mathrm{A}_{0}\right]$. 